\title{
Hibernating switched current cells for low power 14-bit Digital to Analog Converters
}

\begin{abstract}
Static power dissipation remains an issue in low power, high speed and high resolution Digital to Analog Converters (DAC). The architecture of such converters often incorporate switched current sources for the higher significant bits of the input signal. In practice, a switched current source is completely turned off when not contributing to generate the analog output and turned on when required. The energy required to reactivate the circuit contribute to static power dissipation. This work proposes a new type of hibernating switched current source that has lower reactivation energy, thus lowers static power dissipation. Simulation shows that the reactivation energy for a 14-bit, $100 \mathrm{MHz}$ DAC utilizing this new type of current cell is only 52.5 pJoules. This allows a low power dissipation of only $25 \mathrm{~mW}$.
\end{abstract}

Keyword: Current steering; Digital to Analog Converter (DAC); Hibernating switched current cell; Power delay product; Static power 